\title{
High Performance Robust Adaptive Beamforming in the Presence of Array Imperfections
}

\author{
Wenxing Li, ${ }^{1}$ Xiaojun Mao, ${ }^{1}$ Zhuqun Zhai, ${ }^{2}$ and Yingsong $\mathrm{Li}^{1}$ \\ ${ }^{1}$ College of Information and Communications Engineering, Harbin Engineering University, Harbin 150001, China \\ ${ }^{2}$ Naval Academy of Armament, Beijing 100161, China \\ Correspondence should be addressed to Xiaojun Mao; wwwmaoxiaojun@126.com
}

Received 18 April 2016; Accepted 8 June 2016

Academic Editor: Shih Yuan Chen

Copyright (c) 2016 Wenxing Li et al. This is an open access article distributed under the Creative Commons Attribution License, which permits unrestricted use, distribution, and reproduction in any medium, provided the original work is properly cited.

\begin{abstract}
A high performance robust beamforming scheme is proposed to combat model mismatch. Our method lies in the novel construction of interference-plus-noise (IPN) covariance matrix. The IPN covariance matrix consists of two parts. The first part is obtained by utilizing the Capon spectrum estimator integrated over a region separated from the direction of the desired signal and the second part is acquired by removing the desired signal component from the sample covariance matrix. Then a weighted summation of these two parts is utilized to reconstruct the IPN matrix. Moreover, a steering vector estimation method based on orthogonal constraint is also proposed. In this method, the presumed steering vector is corrected via orthogonal constraint under the condition where the estimation does not converge to any of the interference steering vectors. To further improve the proposed method in low signal-to-noise ratio (SNR), a hybrid method is proposed by incorporating the diagonal loading method into the IPN matrix reconstruction. Finally, various simulations are performed to demonstrate that the proposed beamformer provides strong robustness against a variety of array mismatches. The output signal-to-interference-plus-noise ratio (SINR) improvement of the beamformer due to the proposed method is significant.
\end{abstract}

\section{Introduction}

Adaptive beamforming is one of the important aspects of array processing, which has been widely used in radar, sonar, mobile communications, radio astronomy, and other fields [1-3]. Beamformers can be regarded as spatial filters, which can enhance the desired signal and suppress the interference effectively. The standard Capon beamformer (SCB), as one of the well-known adaptive beamformers, has excellent resolution and interference rejection capability. However, the SCB is sensitive to the model mismatches, especially, when the signal of interest (SOI) is presented in the training data [4-7]. In some applications like passive sonar and wireless communications, the training data usually contains the SOI. Thus, the performance of the adaptive beamformers may degrade significantly in the presence of array imperfections.

To improve the robustness of adaptive beamformers, many robust adaptive beamforming methods have been developed over the past several decades [8-12]. In [8], a diagonal loading method is proposed to improve the robustness of array against array steering vector (ASV) and covariance matrix mismatches. However, it does not provide any guidance to select the optimal diagonal loading factor. In [9], a robust adaptive beamforming scheme is obtained which aims to cope with the worst-case performance optimization, where the array steering vector is assumed to lie in an uncertain ellipsoidal set. It has been shown that this beamformer coping with the worst-case belongs to a kind of diagonal loading techniques, where the optimal diagonal loading factor can be adjusted according to the ellipsoidal uncertainty set. The robustness of the worst-case beamformer has been greatly improved compared with the simplest diagonal loading method. However, the performance of the worst-case beamformer is mainly determined by the uncertain parameter set, and the uncertainty of the ASV mismatch is difficult to be known accurately in practice.

Robust adaptive beamforming based on steering vector estimation has been proposed in [10]. To estimate the steering vector, one needs to maximize the beamformer output power 
and guarantee the ASV does not converge to any interference steering vectors or their linear combinations, which is a quadratically constrained quadratic programming (QCQP) problem and can be converted to semidefinite programming (SDP). Certainly, the global optimal solution can be found efficiently.

Recently, robust adaptive beamforming based on interference-plus-noise (IPN) covariance matrix reconstruction and ASV estimation has been proposed in [11]; the IPN covariance matrix was reconstructed by utilizing the Capon spectrum to integrate over a region separated from the SOI direction. This method can achieve good performance in the case of ASV direction error. However, this method is ineffective in the presence of array calibration errors, especially in low signal-to-noise ratios (SNRs) [12, 13]. In [14], a modified method to reconstruct the IPN covariance matrix has been given, where an annulus uncertainty set is used to constrain the steering vectors of the interference. Then integrate the Capon spectrum over the surface of the annulus, by which the reconstructed IPN matrix without containing the SOI can be obtained. Compared with method in [11], this method can reduce the sensitivity of beamformers to array calibration errors, but the performance improvement is limited, especially at low SNRs.

In this paper, we propose a novel IPN covariance matrix reconstruction method. The estimated IPN covariance matrix consists of two parts. The first part is obtained by utilizing the method proposed in [11]. The second part is obtained by removing the SOI component from the sample covariance matrix, where the SOI component is estimated through eigendecomposition of the sample covariance matrix. Then a weighted summation of two parts is used to reconstruct the IPN matrix, and the weighting parameter is related to the desired signal energy compared with the interference energy. The detailed investigation of the parameters is also provided. To further improve the performance of the proposed method in low SNR, a hybrid method is proposed by using the diagonal loading method in the IPN matrix reconstruction to overcome overestimation of the signal subspace. In order to estimate the actual ASV of the desired signal, the presumed steering vector of SOI is subsequently corrected by using the orthogonal constraint. The estimated ASV is enforced to keep orthogonal to the noise subspace, while avoiding convergence to any of the interference steering vectors. That means the estimated ASV can only converge to the actual ASV of the desired signal.

Simulation results show that the output signal-to-interference-plus-noise ratio (SINR) of the proposed adaptive beamforming is closer to the optimal value than other previously developed robust beamforming methods in the presence of various array imperfections, especially when the array calibration error exists. Performance improvement due to the proposed method approach is significant.

\section{The Signal Model}

We consider a uniform linear array (ULA) with $N$ unidirectional antennas with spacing $d$. We assume that there are
$M+1$ signals arriving from the directions $\theta_{p}, p=0,1, \ldots, M$. The received data of the array $\mathbf{X}(k)$ can be expressed as

$$
\mathbf{X}(k)=\mathbf{A S}(k)+\mathbf{N}(k),
$$

where $\mathbf{X}(k)=\left[x_{1}(k), x_{2}(k), \ldots, x_{N}(k)\right]^{T}$ is $N \times 1$ array observations data vector. $k$ is the time index. $\mathbf{S}(k)=\left[s_{0}(k), s_{1}(k)\right.$, $\left.\ldots, s_{M}(k)\right]^{T}$, and $s_{p}(k)$ denotes the complex waveform of the $p$ th signal. Here, $s_{0}(k)$ is considered as the SOI, while $s_{i}(k), i=1, \ldots, M$, are the interference. $\mathbf{N}(k)=\left[n_{1}(k), n_{2}(k)\right.$, $\left.\ldots, n_{N}(k)\right]^{T}$ is a vector of the additive white sensor noise, $\mathbf{A}=\left[\mathbf{a}\left(\theta_{0}\right), \mathbf{a}\left(\theta_{1}\right), \ldots, \mathbf{a}\left(\theta_{M}\right)\right]$, where $\mathbf{a}\left(\theta_{p}\right)=\left[1, e^{j \beta_{p}}, \ldots\right.$, $\left.e^{j(N-1) \beta_{p}}\right]^{T}$ represents a steering vector in the $\theta_{p}$ direction, and $\beta_{p}$ is the wave number that can be represented as $\beta_{p}=$ $2 \pi d \sin \left(\theta_{p}\right) / \lambda$.

We assume that the signal and noise are statistically independent. The output of the beamformer $y(k)$ is given by

$$
y(k)=\mathbf{w}^{H} \mathbf{X}(k),
$$

where $\mathbf{w}$ is the $N \times 1$ optimal weight vector.

The minimum variance distortionless response (MVDR) beamformer is formulated as the following linearly constrained quadratic optimization problem:

$$
\begin{aligned}
\min _{\mathbf{w}} & \mathbf{w}^{H} \mathbf{R}_{i+n} \mathbf{w} \\
\text { subject to } & \mathbf{w}^{H} \overline{\mathbf{a}}_{0}=1,
\end{aligned}
$$

where $\overline{\mathbf{a}}_{0}$ is the presumed ASV of the SOI, $\mathbf{w}$ is the optimal weight vector, and $\mathbf{R}_{i+n}$ is the IPN covariance matrix. In practice, $\mathbf{R}_{i+n}$ is commonly replaced by the sample covariance matrix

$$
\widehat{\mathbf{R}}=\frac{1}{K} \sum_{k=1}^{K} \mathbf{X}(k) \mathbf{X}(k)^{H},
$$

where $K$ is the number of snapshots. Thus, the optimal solution to (3) is

$$
\mathbf{w}=\frac{\widehat{\mathbf{R}}^{-1} \overline{\mathbf{a}}_{0}}{\overline{\mathbf{a}}_{0}^{H} \widehat{\mathbf{R}}^{-1} \overline{\mathbf{a}}_{0}} .
$$

The solution (5) is commonly referred to as the sample matrix inverse (SMI). The output SINR of the beamformer is defined as

$$
\operatorname{SINR}=\frac{\sigma_{0}^{2}\left|\mathbf{w}^{H} \mathbf{a}_{0}\right|^{2}}{\mathbf{w}^{H} \mathbf{R}_{i+n} \mathbf{w}},
$$

where $\sigma_{0}^{2}$ is the power of the desired signal and $\mathbf{a}_{0}$ is the actual ASV of the desired signal. The standard MVDR beamformer can produce sharp nulls at the direction of interference with a good interference rejection performance and high output SINR in the ideal case. However, in practice, the knowledge of ASVs can be imprecise, which means the mismatch may exist between the presumed and actual ASVs. In such a case, the standard MVDR beamformer may attempt to suppress 
the desired signal as it was interference. The performance of the beamformer will degrade seriously.

Recently, in [11], the authors proposed a robust beamforming technique by reconstructing the IPN covariance matrix, which uses the following Capon spectrum as an estimate of the spatial power spectrum over all possible directions [15]. It is well known that the Capon spatial spectrum estimator is

$$
\widehat{P}(\theta)=\frac{1}{\mathbf{a}^{H}(\theta) \widehat{\mathbf{R}}^{-1} \mathbf{a}(\theta)},
$$

where $\mathbf{a}(\theta)$ is the ASV associated with direction that has the structure defined by the antenna array geometry. There are also other candidate spatial spectrum estimators except (7). Using the Capon spatial spectrum, the IPN covariance matrix $\widehat{\mathbf{R}}_{i+n}$ can be reconstructed as

$$
\begin{aligned}
\widehat{\mathbf{R}}_{i+n} & =\int_{\bar{\Theta}} \widehat{P}(\theta) \mathbf{a}(\theta) \mathbf{a}^{H}(\theta) d \theta \\
& =\int_{\bar{\Theta}} \frac{\mathbf{a}(\theta) \mathbf{a}^{H}(\theta)}{\mathbf{a}^{H}(\theta) \widehat{\mathbf{R}}^{-1} \mathbf{a}(\theta)} d \theta
\end{aligned}
$$

where $\bar{\Theta}$ is the complement sector of $\Theta$ in the whole spatial domain and $\Theta$ is an angular sector in which the desired signal may be located. It can be observed from (8) that $\widehat{\mathbf{R}}_{i+n}$ is obtained by collecting the information of interference and noise in the sector $\bar{\Theta}$, and the desired signal was not included in it as long as the direction of SOI is located in $\Theta$. That means that the effect of the desired signal has been removed from the reconstructed covariance matrix, and thus superior performance can be provided compared with the existing robust beamforming method in the case of ASV errors. However, this method is based on the premise that the precise information about the array structure is known exactly in advance, which is almost impossible in practice. As a consequence, the method in [11] will be ineffective in the presence of array calibration errors, such as the gain and phase perturbations and antenna location error. The performance of this method degraded seriously in the case of array calibration error, especially in low input SNRs $[12,13]$.

\section{The Proposed Algorithm}

In this section, we propose a novel method to reconstruct the IPN matrix. The estimated IPN matrix consists of two parts. The first part is $\widehat{\mathbf{R}}_{i+n}$ which is expressed as in (8). As for the second part, the desired signal component is estimated from the eigenvectors of the sample covariance matrix $\widehat{\mathbf{R}}$, and the desired signal can be removed from $\widehat{\mathbf{R}}$. Thus, the rest of the covariance matrix can be regarded as the second part of the estimated IPN matrix. A weighted summation of two parts is used to reconstruct the IPN matrix, and the weighting parameter is used to reflect the desired signal energy compared with the interference energy. With the reconstructed IPN matrix, the presumed ASV of the desired signal is subsequently corrected by solving the QCQP problem.
3.1. The Basic Proposed Beamformer. The sample covariance matrix $\widehat{\mathbf{R}}$ defined by (4) can be decomposed as

$$
\widehat{\mathbf{R}}=\sum_{i=1}^{N} \lambda_{i} \boldsymbol{v}_{i} \boldsymbol{v}_{i}^{H}=\mathbf{U}_{s} \Lambda_{s} \mathbf{U}_{S}^{H}+\mathbf{U}_{n} \Lambda_{n} \mathbf{U}_{n}^{H},
$$

where $\lambda_{i}, i=1, \ldots, N$, are the eigenvalues of $\widehat{\mathbf{R}}$ and $\boldsymbol{v}_{i}, i=$ $1, \ldots, N$, are the corresponding eigenvectors. $\mathbf{U}_{s}=\left[\boldsymbol{v}_{1}, \boldsymbol{v}_{2}\right.$, $\left.\ldots, v_{M+1}\right]$ represents the signal-plus-interference (SPI) subspace, which is formed by the $M$ interference plus a SOI. $\mathbf{U}_{n}=$ $\left[\boldsymbol{v}_{M+2}, \boldsymbol{v}_{M+3}, \ldots, \boldsymbol{v}_{N}\right]$ represents the noise subspace, $\Lambda_{s}=$ $\operatorname{diag}\left\{\lambda_{1}, \lambda_{2} \ldots, \lambda_{M+1}\right\}$ are the eigenvalues of the SPI, and $\Lambda_{n}=\operatorname{diag}\left\{\lambda_{M+2}, \lambda_{M+3}, \ldots, \lambda_{N}\right\}$ are the eigenvalues of noise. As we know, the eigenvectors of $\mathbf{U}_{s}$ and the ASV s of the SPI lie in the same subspace. What is more, the mismatch between $\mathbf{a}_{0}$ and $\overline{\mathbf{a}}_{0}$ is not too large in fact. We project the presumed ASV of the SOI $\overline{\mathbf{a}}_{0}$ onto the eigenvectors to get $p(i), i=1,2, \ldots, N$. $p(i)$ can be expressed as

$$
p(i)=\left|\boldsymbol{v}_{i}^{H} \overline{\mathbf{a}}_{0}\right|^{2} \quad(i=1,2, \ldots, N) .
$$

The projections $p(i)$ can be arranged in descending order, as $p_{[N]} \geq p_{[N-1]} \geq \cdots \geq p_{[1]}$. Meanwhile, the corresponding eigenvectors can be arranged as $\boldsymbol{v}_{[N]}, \boldsymbol{v}_{[N-1]}, \ldots, \boldsymbol{v}_{[1]}$, and the corresponding eigenvectors can be arranged as $\lambda_{[N]}, \lambda_{[N-1]}, \ldots, \lambda_{[1]}$. It is important to note that the eigenvectors $\boldsymbol{v}_{[N]}, \boldsymbol{v}_{[N-1]}, \ldots, \boldsymbol{v}_{[1]}$ and the corresponding eigenvalues $\lambda_{[N]}, \lambda_{[N-1]}, \ldots, \lambda_{[1]}$ are the same as in (9), but they have been reordered according to $p_{[N]} \geq p_{[N-1]} \geq \cdots \geq p_{[1]}$.

As well known, the maximum of the projections $p_{[N]}$ is obtained when $\boldsymbol{v}_{i}$ is the eigenvector corresponding to the SOI [12]. That means the eigenvector corresponding to the SOI is determined according to the projections $p(i), i=1,2, \ldots, N$. It is easy to see that $\boldsymbol{v}_{[N]}$ is the eigenvector of SOI and $\lambda_{[N]}$ is the corresponding eigenvalue. We remove $\boldsymbol{v}_{[N]}$ and $\lambda_{[N]}$ from $\widehat{\mathbf{R}}$ and obtain $\overline{\mathbf{U}}_{i+n}=\left[\boldsymbol{v}_{[N-1]}, \boldsymbol{v}_{[N-2]}, \ldots, \boldsymbol{v}_{[1]}\right]$. Then, we have

$$
\overline{\mathbf{R}}_{i+n}=\overline{\mathbf{U}}_{i+n} \overline{\mathbf{U}}_{i+n}^{H} .
$$

Since the SOI component has been removed from $\widehat{\mathbf{R}}, \overline{\mathbf{R}}_{i+n}$ can be regarded as a IPN matrix. We now propose a new method to estimate the IPN matrix by using the weighted combination as follows:

$$
\widetilde{\mathbf{R}}_{i+n}=\alpha \widehat{\mathbf{R}}_{i+n}+\beta(1-\alpha) \overline{\mathbf{R}}_{i+n},
$$

where $\alpha=\lambda_{[N]} /\left(\lambda_{[1]}+\lambda_{[2]}+\cdots+\lambda_{[N-1]}+\lambda_{[N]}\right)$ and $\beta=$ $\lambda_{[1]}+\lambda_{[2]}+\cdots+\lambda_{[N-1]}$. We can see that the parameter $\alpha \in[0,1]$, which can reflect the SOI energy compared with the interference energy. The parameter $\alpha$ is used to adjust the proportion of $\widehat{\mathbf{R}}_{i+n}$ and $\overline{\mathbf{R}}_{i+n}$ according to the input SNR. When the input SNR is high, $\alpha$ is close to 1 , and $\widetilde{\mathbf{R}}_{i+n}$ is mainly composed of $\widehat{\mathbf{R}}_{i+n}$. When the input SNR is low, $\alpha$ is close to 0 , and $\widetilde{\mathbf{R}}_{i+n}$ is mainly composed of $\overline{\mathbf{R}}_{i+n}$.

Since the actual steering vector of the desired signal is difficult to obtain in practical applications and the mismatches between the presumed and actual ASVs cause significant performance degradation, here, we propose a new ASV 
estimation method based on the orthogonal constraint. As we all know, the actual ASVs of the signal and interference should be orthogonal to the noise subspace, which means that we can obtain accurate ASV of the desired signal by using orthogonal constraint under the condition where the estimate does not converge to any of the interference steering vectors. Taking into consideration the mismatch norm constraint, the problem of estimating the ASV of the desired signal based on orthogonal constraint can be formulated according to the following optimization problem:

$$
\begin{array}{cl}
\min _{\widetilde{\mathbf{a}}_{0}} & \widetilde{\mathbf{a}}_{0}^{H} \mathbf{U}_{n} \mathbf{U}_{n}^{H} \widetilde{\mathbf{a}}_{0} \\
\text { subject to } & \widetilde{\mathbf{a}}_{0}^{H} \widetilde{\mathbf{C}}^{\widetilde{\mathbf{a}}_{0} \leq \Delta_{0}} \\
& \left\|\widetilde{\mathbf{a}}_{0}-\overline{\mathbf{a}}_{0}\right\|^{2} \leq \varsigma,
\end{array}
$$

where $\widetilde{\mathbf{a}}_{0}$ is the corrected ASV, $\widetilde{\mathbf{C}}=\int_{\bar{\Theta}} \mathbf{a}(\theta) \mathbf{a}^{H}(\theta) d \theta, \Delta_{0}=$ $\max _{\theta \in \Theta} \mathbf{a}(\theta) \widetilde{\mathbf{C}} \mathbf{a}^{H}(\theta),\|\cdot\|$ denotes the Euclidean norm, and $\varsigma$ is the norm bound of the mismatches. The objective function (13) keeps the estimated $\widetilde{\mathbf{a}}_{0}$ within the signal or interference limits, while the constraints (14) and (15) can guarantee that $\widetilde{\mathbf{a}}_{0}$ does not converge to any of the interference steering vectors. Thus, the accurate ASV of the desired signal can be obtained.

This optimization problem can be efficiently solved by convex optimization toolbox [16]. Finally, with the corrected ASV $\widetilde{\mathbf{a}}_{0}$ and estimated IPN matrix $\widetilde{\mathbf{R}}_{i+n}$, the proposed beamformer weighting vector can be calculated as

$$
\widetilde{\mathbf{w}}=\frac{\widetilde{\mathbf{R}}_{i+n}^{-1} \widetilde{\mathbf{a}}_{0}}{\widetilde{\mathbf{a}}_{0}^{H} \widetilde{\mathbf{R}}_{i+n}^{-1} \widetilde{\mathbf{a}}_{0}} .
$$

3.2. The Improvement of Basic Beamformer. As we all know, the signal and noise subspaces of the eigencomposition cannot be accurately separated in practice, especially when the SNR is low. As a result, the performance of the basic proposed beamformer is not so good when SNR $\leq-10 \mathrm{~dB}$ due to the overestimation of signal subspace, which is an inherent shortcoming of the subspace decomposition. However, the LSMI, worst-case, and SDP-RAB perform almost equivalently when $\mathrm{SNR} \leq-10 \mathrm{~dB}$. That is to say, we prefer to use LSMI method in low SNR due to its low computational complexity and good performance.

We can use the parameter $\gamma$ to reflect the input SNR directly, which can be expressed as

$$
\gamma=10 \log \left(\frac{\lambda_{[N]}}{\lambda_{[1]}}\right) .
$$

As we all know, if the input SNR is very small, $\gamma<0$, whereas, in high SNR situations, the large value of $\gamma$ can be achieved. Here, we give an example to discuss the relationship between $\gamma$ and the input SNR.

We consider a ULA of $N=10$ antennas spaced at a half wavelength distance. Additive noise is modeled as independent complex Gaussian noise with zero mean and unit variance. Two independent interference vectors are from

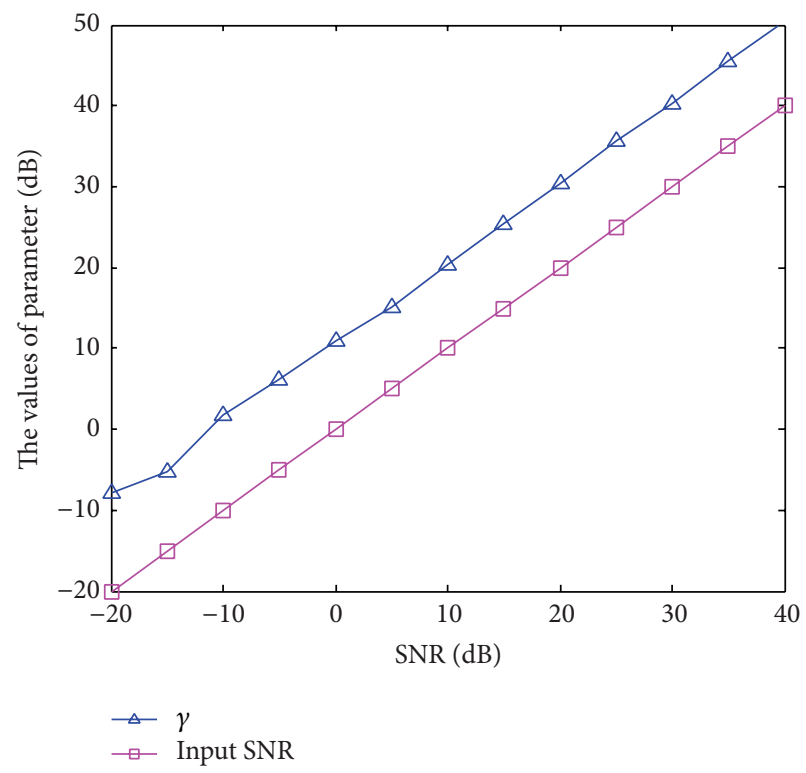

Figure 1: Values of $\gamma$ versus the SNRs.

the directions of $30^{\circ}$ and $50^{\circ}$, respectively. The interferenceto-noise ratios (INRs) of the interference are $20 \mathrm{~dB}$ unless otherwise is specified. The sample covariance matrix is collected based on $K=50$ data snapshots. The SOI is assumed from the direction of $3^{\circ}$. The possible angular sector of the SOI is set to $\Theta=\left[-5^{\circ}, 11^{\circ}\right]$, so the complement sector is $\bar{\Theta}=$ $\left[-90^{\circ},-5^{\circ}\right) \cup\left(11^{\circ}, 90^{\circ}\right]$ and the parameter $\varsigma=1$. The difference between the presumed and actual positions of each antenna element is modeled as a uniform random variable distributed in the interval $[-0.075 \lambda, 0.075 \lambda]$, where $\lambda$ represents the wavelength. The actual DOA of SOI is $5^{\circ}$, which means the DOA mismatch is $2^{\circ}$. Figure 1 shows the values of $\gamma$ versus input SNRs.

We can observe from Figure 1 that the trend of $\gamma$ agrees well with the input SINR when the input SNR $>-10 \mathrm{~dB}$. The relationship between $\gamma$ and SNR is almost linear. We can see that the parameter $\gamma$ can reflect the change of input SNR accurately as long as SNR is large enough. That means $\lambda_{[N]}$ and $\boldsymbol{v}_{[N]}$ are the actual eigenvalue and eigenvector of the desired signal, respectively. Certainly, the rest of the sample covariance matrix $\overline{\mathbf{U}}_{i+n}$ can be estimated as the IPN matrix. When SNR $<-10 \mathrm{~dB}, \gamma$ failed to reflect the input SNR due to the overestimation of signal subspace. Luckily, the traditional diagonal loading method can achieve the same performance as other methods in low SNRs. Thus, the basic proposed IPN reconstruction method can be extended as

$$
\widetilde{\mathbf{R}}_{i+n}= \begin{cases}\alpha \widehat{\mathbf{R}}_{i+n}+\beta(1-\alpha) \overline{\mathbf{R}}_{i+n} & \gamma \geq 0 \\ \widehat{\mathbf{R}}+\eta \mathbf{I} & \gamma<0,\end{cases}
$$

where $\mathbf{I}$ is an identity matrix and $\eta$ is the diagonal loading factor. Since $\widehat{\mathbf{R}}+\eta \mathbf{I}$ is only used in low SNRs, we can set $\eta$ as twice the noise power to obtain good performance. The improved proposed method can overcome the problem of performance degradation in low SNRs. Figure 2 shows the output SINRs versus input SNRs. 


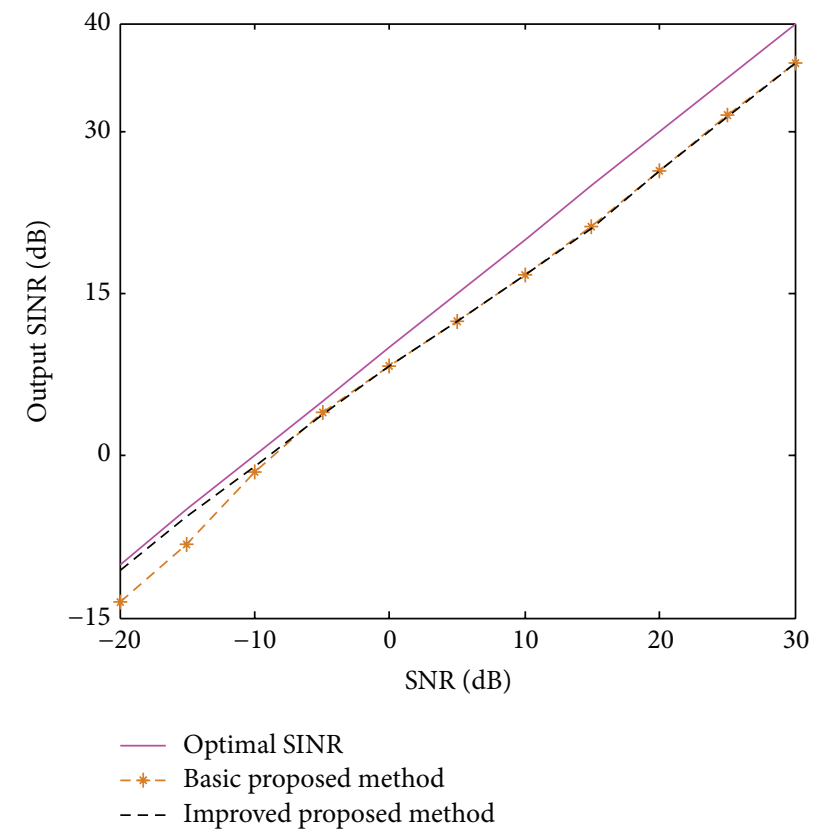

FIGURE 2: Output SINR of beamformers versus input SNR for the case of perturbations in antenna array geometry.

We can see that the improved reconstruction method outperforms the original method significantly in low SNRs, which can get the high output SINR performance in both of them at low and high SNR.

The main computational complexity of the proposed method is the IPN matrix reconstruction problem and the QCQP problem. The computational complexity of the former is $O\left(S N^{2}\right)$, where $S$ is the number of sampling points in $\bar{\Theta}$ during the reconstruction of covariance matrix. Generally, $S \gg N$. The latter can be efficiently obtained using interior point methods, which has complexity of $O\left(N^{3.5}\right)$. Consequently, the overall complexity of our beamformer is $O\left(S N^{2}\right)$. The LSMI algorithm has a complexity of $O\left(N^{3}\right)$. The worstcase beamforming and the beamformer of [10] (semidefinite programming robust adaptive beamforming (SDP-RAB)) have at least the complexity of $O\left(N^{3.5}\right)$. Generally speaking, the proposed method has the same complexity with the beamformer of [11] and beamformer of [14] but it can provide significantly more robust performance.

\section{Simulation Results}

In this section, the basic simulation conditions are the same as above unless otherwise is specified. The proposed beamformer is investigated and compared with the diagonally loaded SMI (LSMI) [8], worst-case beamformer [9], the SDP$\mathrm{RAB}$, and the beamformer of [11]. The optimal parameter $\varepsilon=0.3 \mathrm{~N}$ is used for the worst-case beamformer, while the diagonal loading factor of LSMI is selected as twice the noise power. In all simulations, 200 Monte Carlo runs are used to obtain each simulated point. CVX software was used to solve these convex optimization problems.

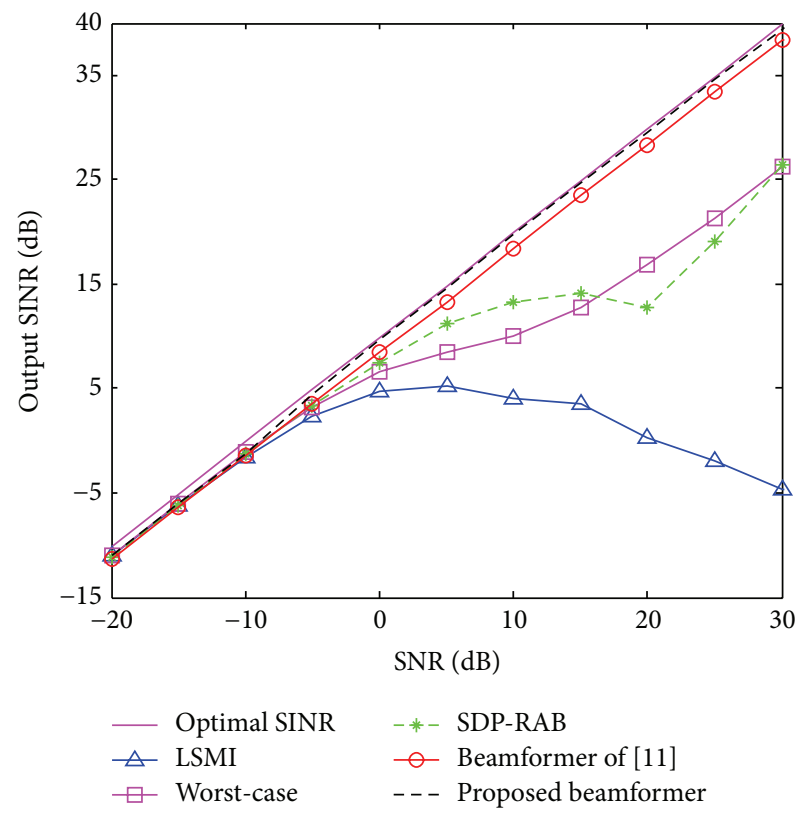

FIgURE 3: Output SINR versus the input SNR in the case of look direction mismatch.

Simulation Example 1 (signal look direction mismatch). In this example, the performance of the proposed beamformer against the random desired signal direction error is investigated. We assume that the random DOA estimation mismatch of the SOI is uniformly distributed in $\left[-4^{\circ}, 4^{\circ}\right]$ for each simulation run. That is to say, the DOA of the signal is uniformly distributed in $\left[-1^{\circ}, 7^{\circ}\right]$. Here, the random DOAs of the desired signal change from run to run but remain fixed from snapshot to snapshot.

Figure 3 displays the mean output SINR of the tested methods versus the SNR for $K=30$. We can find that the LSMI, worst-case, and SDP-RAB suffer a significant performance degradation in high SNR due to the presence of desired signal component. It can be clearly seen that the proposed beamformer outperforms the other beamformers. The performance of the proposed beamformers is always close to the optimal SINR in a large range from -20 to $30 \mathrm{~dB}$. In particular, it can be observed that the output SINR of proposed beamformer is about 1.1 dB higher than beamformer of [11] at $\mathrm{SNR}=10$, and the deviation from the optimal is only $0.3 \mathrm{~dB}$. In low SNR, the performance of the proposed beamformer is the almost the same as the LSMI due to the same covariance matrix.

Figure 4 displays the mean output SINR against the number of snapshots for the fixed input SNR $=10 \mathrm{~dB}$. We can observe that the proposed beamformer enjoys higher output SINR compared with other beamformers when the number of snapshots is larger than 20. The inaccurate estimation of the SOI component in $\widehat{\mathbf{R}}$ is the main reason why the proposed beamformer suffers performance degradation when the number of snapshots is very small. Generally speaking, the number of snapshots is larger than 20 in most of the practical applications. 


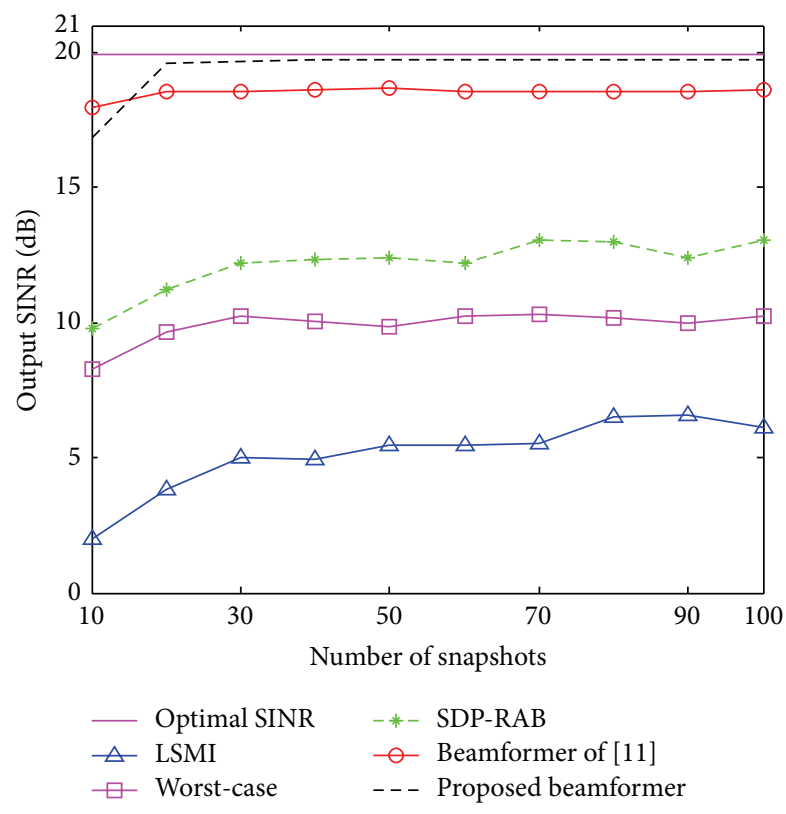

FIGURE 4: Output SINR versus the number of snapshots in the case of look direction mismatch.

Simulation Example 2 (desired ASV mismatch due to wavefront distortion). In this simulation, we consider that the desired signal ASV is distorted by the effects of wave propagation in an inhomogeneous medium which is used in [10]. In particular, the independent-increment phase distortions are accumulated from the components of the presumed ASV. Assume that the phase increments are fixed in each simulation runs and are independently chosen from a Gaussian random generator with zero mean and standard deviation 0.04 . Figure 5 shows the output SINR of the beamformers versus input SNR for $K=30$. Figure 6 shows the output SINR of the beamformers versus the number of snapshots for fixed input $\mathrm{SNR}=10 \mathrm{~dB}$.

It can be observed from these figures that the output SINR of LSMI decreased sharply with an increment of the input SNR. The proposed beamformer enjoys best performance compared to other beamformers in the case of wavefront distortion in the whole $\mathrm{SNR}$ range. In particular, the deviation from the optimal is only $0.3 \mathrm{~dB}$ when $\mathrm{SNR}=10 \mathrm{~dB}$. As described above, the performance of the proposed method degraded in small number of snapshots due to the inaccurate estimation of the SOI component.

Simulation Example 3 (desired ASV mismatch due to coherent local scattering). In this simulation, we consider that the desired ASV is distorted by local scattering effects. The actual ASV is formed by several signal paths, which can be modeled as

$$
\mathbf{a}=\overline{\mathbf{a}}_{0}+\sum_{i=1}^{T} \exp \left(j \varphi_{i}\right) \mathbf{a}\left(\theta_{i}\right),
$$

where $\overline{\mathbf{a}}_{0}$ denotes the direct path and $T$ represent the number of coherently scattered paths. The $i$ th path $\mathbf{a}\left(\theta_{i}\right)$ is modeled as

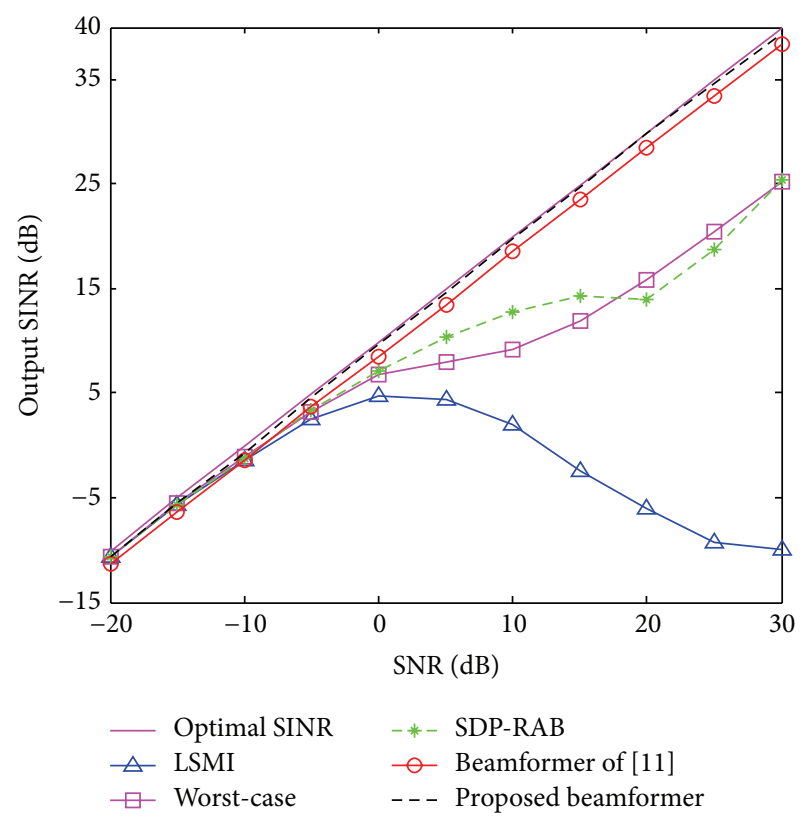

FIGURE 5: Output SINRs versus input SNR in the case of wavefront distortion.

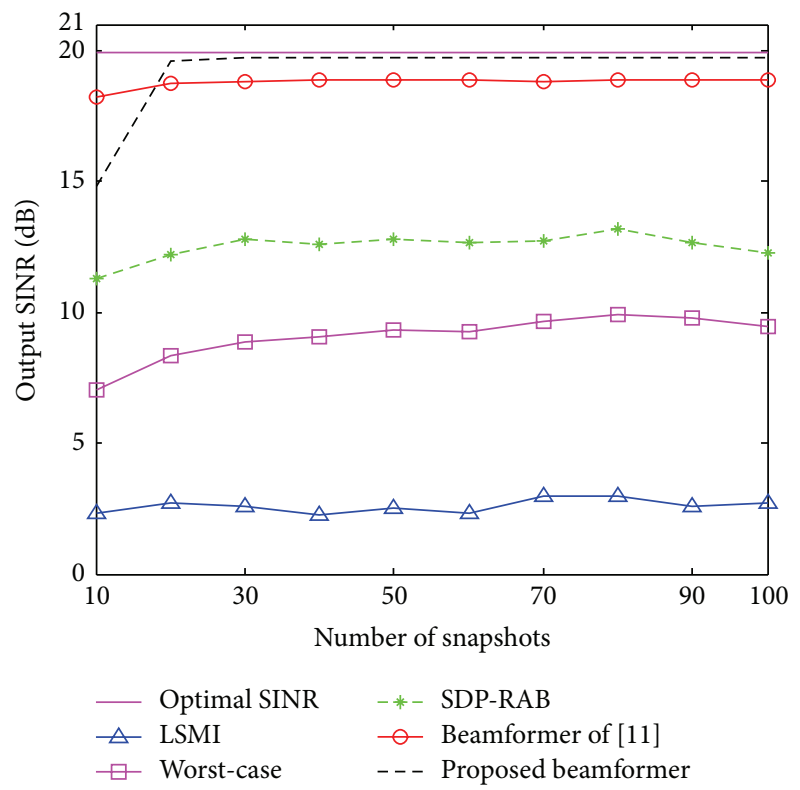

FIGURE 6: Output SINRs versus the number of snapshots in the case of wavefront distortion.

a plane wave which is incident on the array at the direction of $\theta_{i}$. The parameter $\varphi_{i}$ represents the path phases that are independently and uniformly drawn from the interval $[0,2 \pi]$ in each simulation run. In this paper, we set $T=4$. $\theta_{i}$ comes from a uniform random generator with mean $3^{\circ}$ and standard deviation $1^{\circ}$. Other simulation parameters are the same as simulation experiment 2 .

Figure 7 shows the output SINR of the beamformers versus input SNR for $K=30$. It can be seen from Figure 7 that the proposed beamformer enjoys highest output SINR 


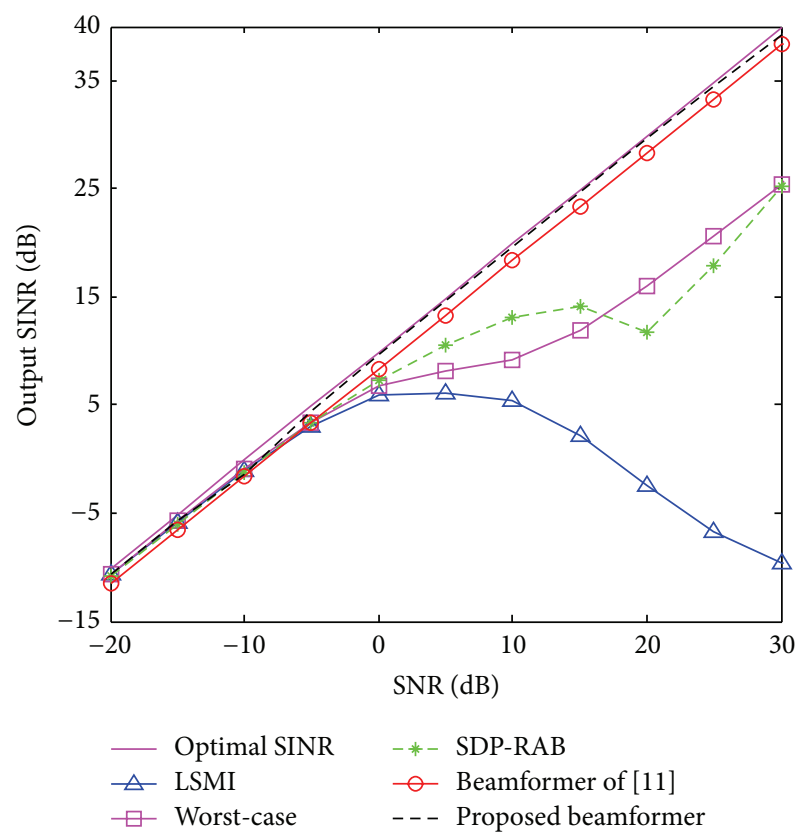

FIGURE 7: Output SINR of beamformers versus input SNR in the case of coherent local scattering.

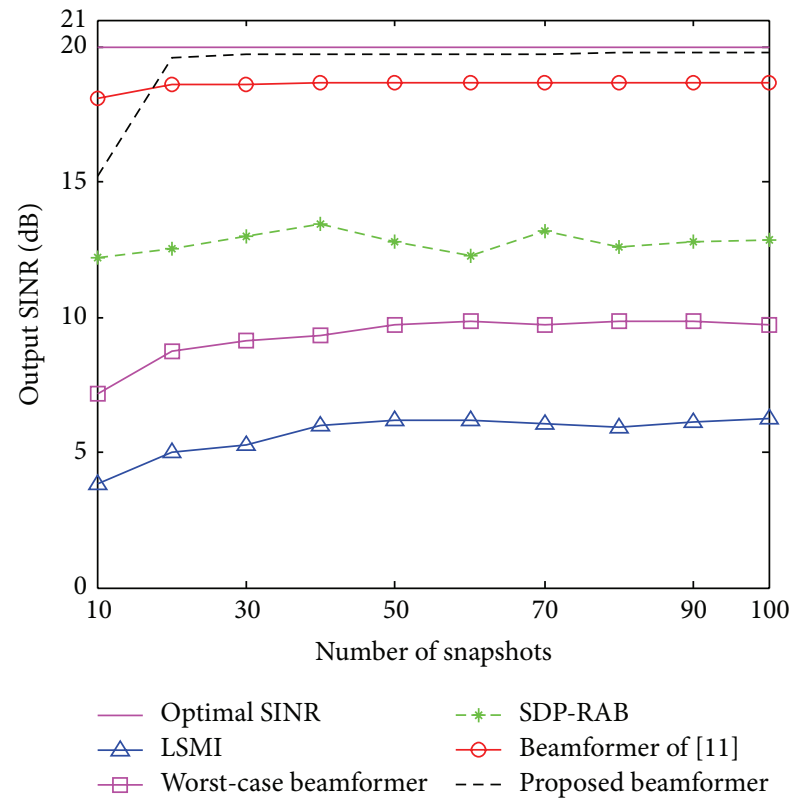

FIGURE 8: Output SINR versus the number of snapshots in the case of coherent local scattering.

performance compared with other beamformers, which is almost always close to the optimal value across a wide range of SNRs. As it can be observed from the figure, the output SINR of the proposed beamformer exceeds beamformer of [11] $1.3 \mathrm{~dB}$ when the input SNR is $10 \mathrm{~dB}$. Figure 8 corresponds to the output SINR performance versus the number of the snapshots with input $\mathrm{SNR}=10 \mathrm{~dB}$. It can be observed that the performance of the proposed beamformer significantly

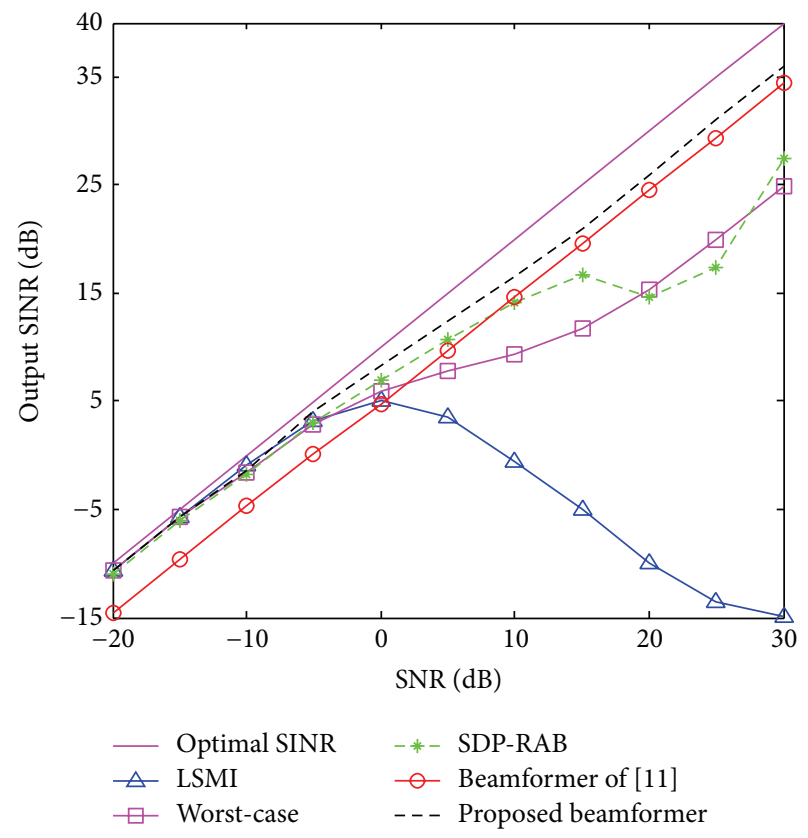

FIGURE 9: Output SINR of beamformers versus input SNR for the case of perturbations in antenna array geometry.

outperforms other beamformers except when the number of the snapshots is smaller than 20.

Simulation Example 4 (effect of the error in the knowledge of the antenna array geometry). In this simulation, we study the effect of the error in the knowledge of antenna array geometry on the performance of the tested beamformer. The difference between the presumed and actual positions of each antenna element is modeled as a uniform random variable distributed in the interval $[-0.075 \lambda, 0.075 \lambda]$, where $\lambda$ represents the wavelength. The actual DOA of SOI is $5^{\circ}$, which means the DOA mismatch is $2^{\circ}$.

Figure 9 shows the output SINR of the beamformers versus input SNR for $K=50$. It can be seen from Figure 9 that beamformer of [11] suffers serious performance degradation in low input SNR due to the inaccurate estimation of the IPN covariance matrix. That means it is ineffective in the presence of array calibration error. In low SNRs, the IPN matrix $\widetilde{\mathbf{R}}_{i+n}$ in our proposed beamformer is mainly composed of $\overline{\mathbf{R}}_{i+n}$, and $\overline{\mathbf{R}}_{i+n}$ is more accurate than $\widehat{\mathbf{R}}_{i+n}$. As a result, the proposed beamformer can provide a significant output SINR improvement over the beamformer of [11]. When the SNRs are high, the IPN matrix $\widetilde{\mathbf{R}}_{i+n}$ is mainly composed of $\widehat{\mathbf{R}}_{i+n}$, and thus the performance of the proposed beamformer is close to the beamformer of [11]. In general, we can observe from Figure 9 that our beamformer achieves almost the best performance when the SNR varies from $-20 \mathrm{~dB}$ to $30 \mathrm{~dB}$. That means the proposed beamformer can provide strong robustness in the presence of the array calibration errors. At $\mathrm{SNR}=10 \mathrm{~dB}$, the output SINR versus the number of snapshots is shown in Figure 10. We can notice that the proposed beamformer still outperforms the other beamformers, only 


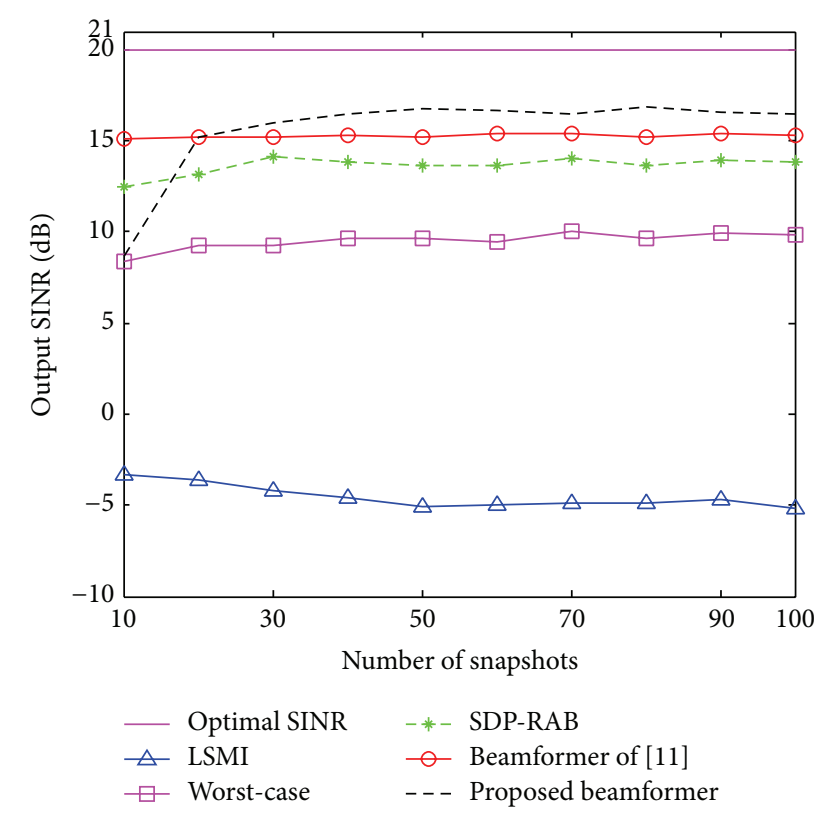

FIGURE 10: Output SINR versus the number of snapshots for the case of perturbations in antenna array geometry.

in the case when the number of the snapshots is smaller than 30 .

Simulation Example 5 (mismatch due to arbitrary ASV errors). In this simulation, we investigate the performance of the proposed beamformer when arbitrary ASV errors are considered. Here, the ASV mismatch is comprehensive and arbitrary-type, which may be caused by direction errors, calibration errors, gain and phase perturbations, and so on. The actual ASVs of are modeled as

$$
\overline{\mathbf{a}}(\theta)=\mathbf{a}(\theta)+\widehat{\mathbf{e}},
$$

where $\overline{\mathbf{a}}(\theta)$ is presumed to be ASVs and $\widehat{\mathbf{e}}$ is a zero mean complex random vector with the variance $\sigma_{e}^{2}$. In this simulation example, all the array imperfections are generated as Gaussian variables with the given variance; $\sigma_{e}^{2}=0.2$.

The output SINR of the beamformers versus input SNR for $K=50$ is displayed in Figure 11. We can notice that the proposed beamformer enjoys an obvious performance improvement compared to the tested beamformers. That means the proposed beamformer is effective against the arbitrary ASV errors. In particular, the output SINR of the proposed beamformer exceeds the beamformer of [14] $2.4 \mathrm{~dB}$ at $\mathrm{SNR}=5 \mathrm{~dB}$. The output SINR versus the number of snapshots for $\mathrm{SNR}=10 \mathrm{~dB}$ is shown in Figure 12. We can notice that the proposed beamformer still outperforms the other beamformers, only in the case when the number of the snapshots is smaller than 30 .

In general, fewer snapshots mean worse performance for certain beamformer, and some algorithms may outperform the proposed method with a small number of snapshots. We can find that the proposed beamformers enjoy the best performance $K \geq 30$. Summarizing, if the errors are only on the ASV associated with the desired signal, meanwhile

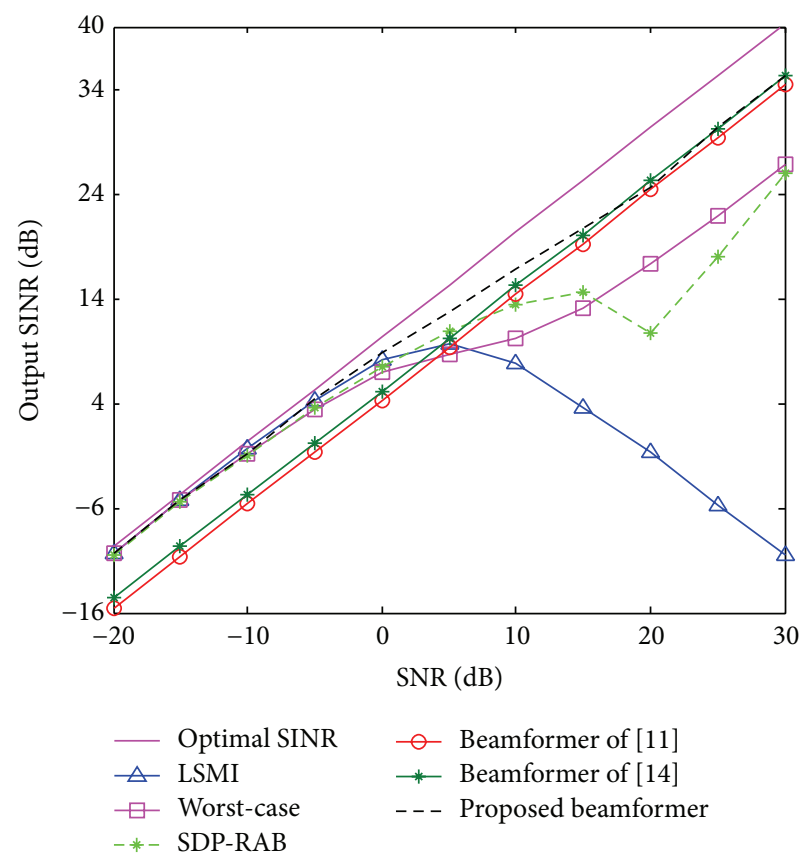

FIGURE 11: Output SINR of beamformers versus input SNR with arbitrary ASV error.

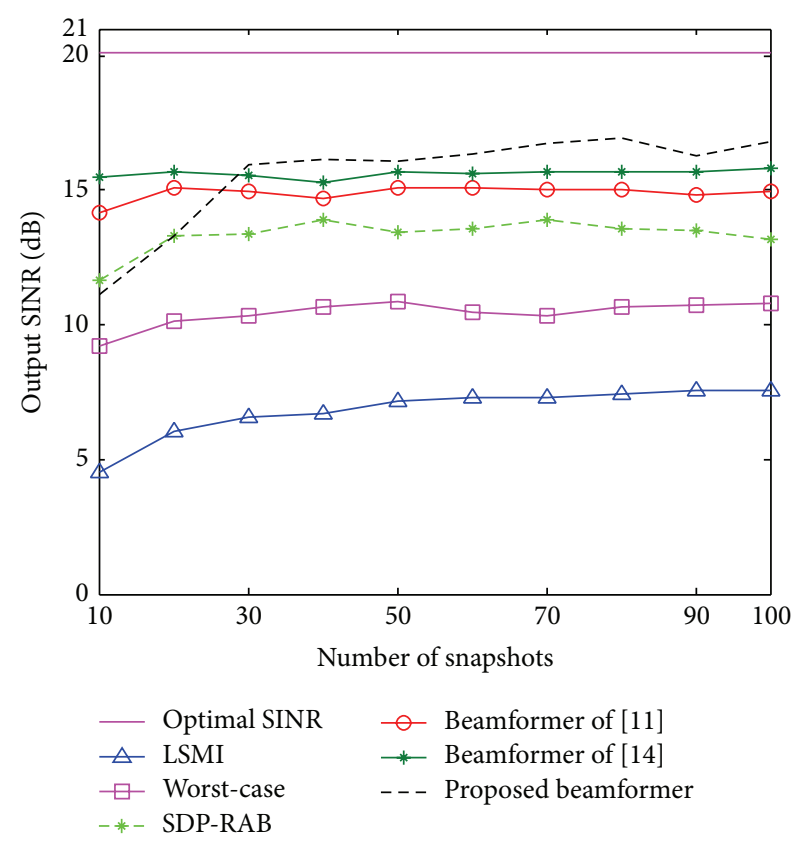

FIGURE 12: Output SINR of beamformers versus snapshots with arbitrary ASV error.

the number of snapshots is very small $(K \leq 30)$; we may prefer to use the beamformer of [11]. Otherwise, the proposed beamformer is suggested; in particular, the array structure information is imprecise.

\section{The Analysis of the Parameters $\alpha$ and $\beta$}

The main idea of the proposed method is to estimate the IPN matrix by using a weighted summation of two parts 


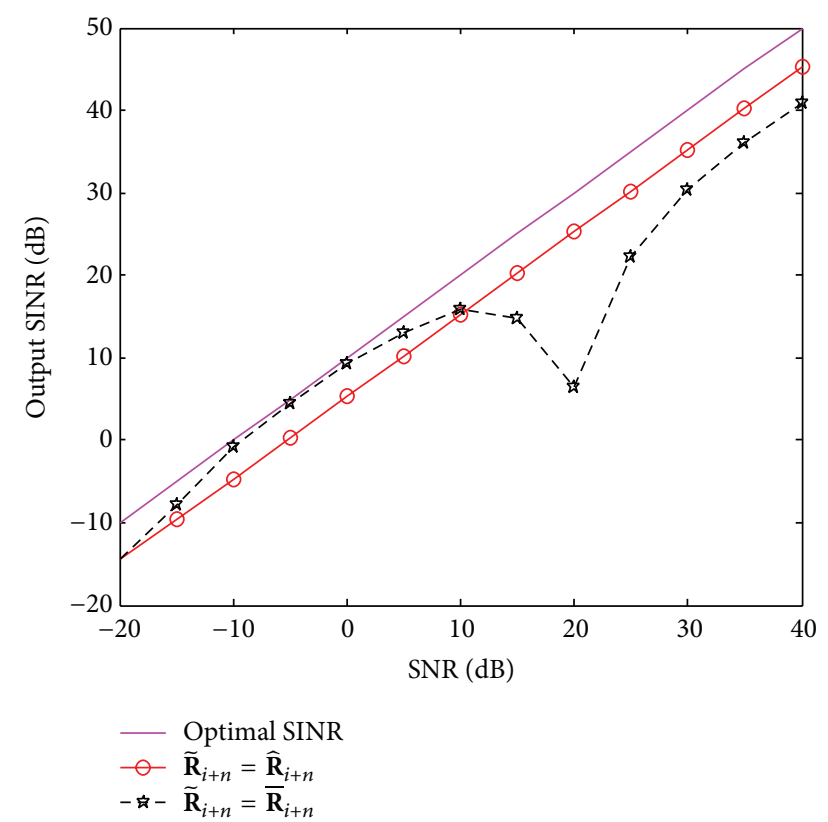

FIGURE 13: Output SINR versus the input SNR with different reconstructed IPN matrixes.

$\left(\widehat{\mathbf{R}}_{i+n}\right.$ and $\left.\overline{\mathbf{R}}_{i+n}\right)$, and the key point is to design proper parameters. We can see that parameters $\alpha$ and $\beta$ are all related to the eigenvalues. Thus, it is a user parameter-free method. In this section, an analysis of the parameters $\alpha$ and $\beta$ as well as some related simulation results is given.

First, we investigate the performance of $\widehat{\mathbf{R}}_{i+n}$ and $\overline{\mathbf{R}}_{i+n}$, respectively. It is worth noting that a very small positive number is added to the diagonal of $\overline{\mathbf{R}}_{i+n}$ to guarantee that $\overline{\mathbf{R}}_{i+n}$ is positive definite.

In this section, we consider the effect of the error in the knowledge of antenna array geometry on the performance of the beamformers. The basic simulation conditions are the same as the simulation experiment 4 unless otherwise is specified. The INR of the interference is $20 \mathrm{~dB}$ unless otherwise is specified.

Figure 13 shows the output SINRs versus input SNRs with different reconstructed IPN matrixes. It can be seen from Figure 13 that $\widehat{\mathbf{R}}_{i+n}$ suffers serious performance degradation in low input SNRs and enjoys good performance in high input SNRs, while $\overline{\mathbf{R}}_{i+n}$ is the opposite. Naturally, it is easy to image that good performance can be obtained by using $\widehat{\mathbf{R}}_{i+n}$ in high SNR, while using $\overline{\mathbf{R}}_{i+n}$ in low SNR, respectively. The difficulty is how to choose the proper proportion of $\overline{\mathbf{R}}_{i+n}$ and $\widehat{\mathbf{R}}_{i+n}$ according to different input SNRs. As we know, the eigenvalues $\left(\lambda_{i}, i=1, \ldots, N\right)$ of the sample covariance matrix $\widehat{\mathbf{R}}$ can reflect the power of signals and noise. However, we can find the eigenvalue corresponding to the SOI $\left(\lambda_{[N]}\right)$ according to the projections $p(i), i=1,2, \ldots, N$, which means we can use $\alpha=\lambda_{[N]} /\left(\lambda_{[1]}+\lambda_{[2]}+\cdots+\lambda_{[N-1]}+\lambda_{[N]}\right)$ to reflect the input SNR indirectly. When the input SNR is high, $\alpha$ is close to 1 and $1-\alpha$ is close to 0 . When the input SNR is low, $\alpha$ is close to 0 and $1-\alpha$ is close to 1 .

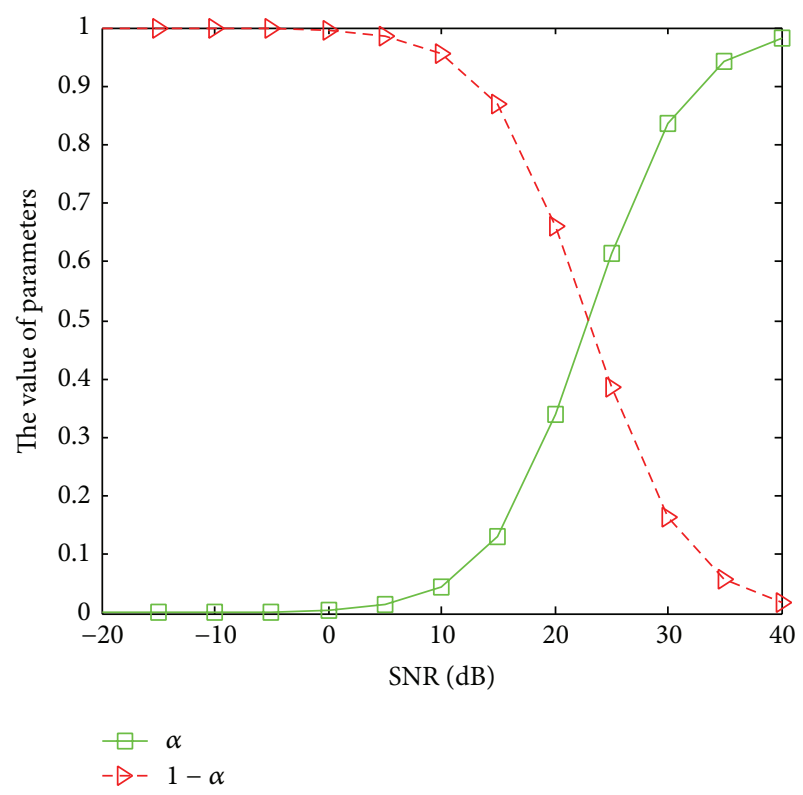

FIgURE 14: Values of $\alpha$ and $1-\alpha$ versus the SNRs.

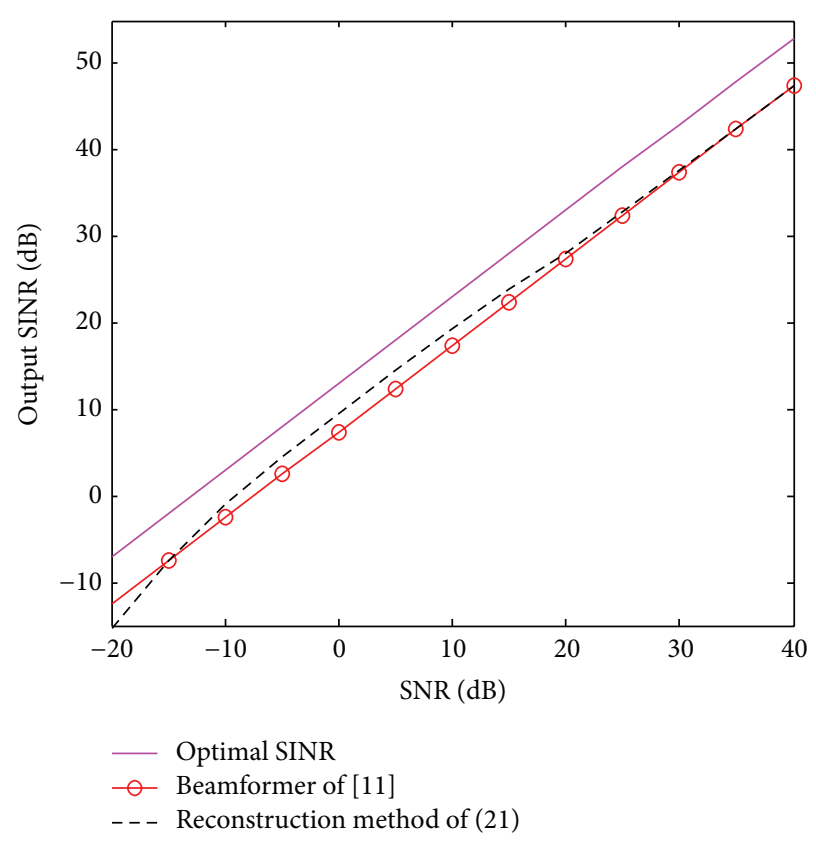

FIGURE 15: Output SINR versus the input SNR with different reconstruction method.

Figure 14 displays the values of $\alpha$ and $1-\alpha$ versus the input SNRs. It can be seen from Figure 14 that our assumption about $\alpha$ is right, so we try to reconstruct the IPN matrix by using

$$
\widetilde{\mathbf{R}}_{i+n}=\alpha \widehat{\mathbf{R}}_{i+n}+(1-\alpha) \overline{\mathbf{R}}_{i+n} .
$$

This is the initial idea. We need to investigate the reconstruction method through the same simulation experiment, and the simulation results were shown in Figure 15.

We can clearly see from Figure 15 that performance of the reconstruction method of (21) is not good enough. The reason 


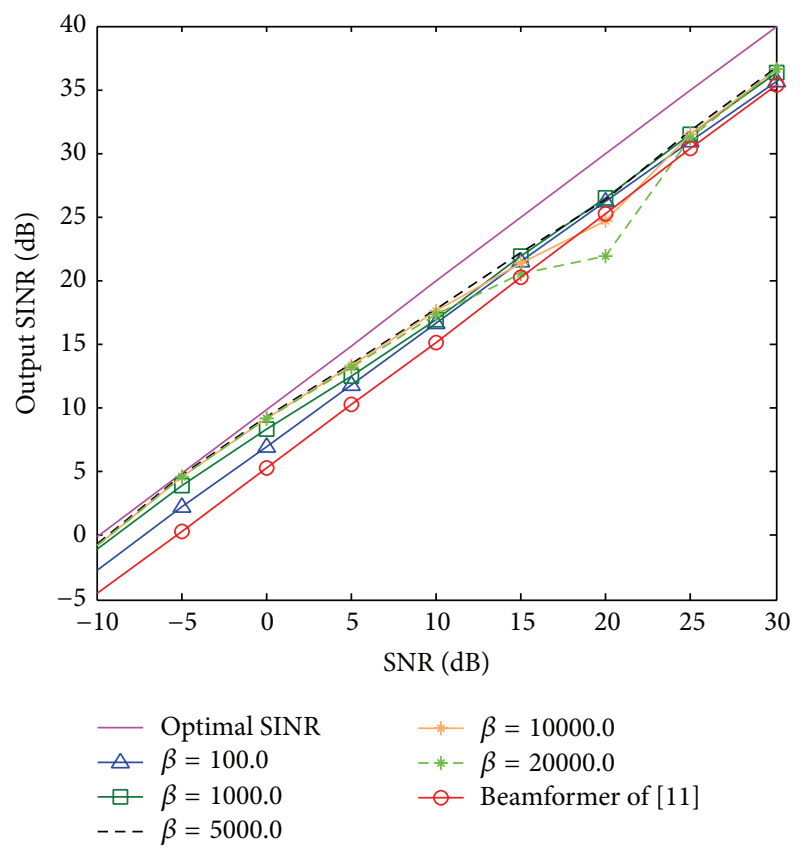

FIgURE 16: Output SINR versus the input SNR with different $\beta$ $(\mathrm{INR}=20 \mathrm{~dB})$.

is that $\overline{\mathbf{R}}_{i+n}$ almost does not work in low SNR. We also can find that $\widehat{\mathbf{R}}_{i+n}$ can work well in high SNR. Thus, we should increase the proportional coefficient of $\overline{\mathbf{R}}_{i+n}$; hence, the IPN matrix is expressed as

$$
\widetilde{\mathbf{R}}_{i+n}=\alpha \widehat{\mathbf{R}}_{i+n}+\beta(1-\alpha) \overline{\mathbf{R}}_{i+n},
$$

where $\beta$ is a positive number. $\beta=100.0, \beta=1000.0, \beta=$ $5000.0, \beta=10000.0$, and $\beta=20000.0$ are used in the simulations. We investigate the reconstruction method through the same experiment as simulation experiment 4 , and the results were shown in Figure 16.

It can be seen from Figure 16 that the performance is not so good in low SNR when $\beta$ is too small $(\beta=100.0$, $\beta=1000.0)$. We also can find that the output SINR decreased significantly at $\mathrm{SNR}=20 \mathrm{~dB}$ when $\beta$ is too large $(\beta=10000.0$, $\beta=20000.0$ ). Obviously, $\beta=5000.0$ enjoys the best performance. We also find that the higher interference power is, the larger $\beta$ is needed, which can be observed from Figures 17 and 18.

Figure 17 shows the output SINRs versus input SNRs with different $\beta$ for INR $=30 \mathrm{~dB}$, and Figure 18 shows the output SINRs versus input SNRs with different $\beta$ for INR $=40 \mathrm{~dB}$. We can see from Figures 17 and 18 that the optimal value of $\beta$ is $\beta=10000.0$ when the INR is $30 \mathrm{~dB}$, and the optimal value of $\beta$ is $\beta=20000.0$ when the INR is $40 \mathrm{~dB}$. This means the optimal value of $\beta$ is positive to the interference power. It is because the higher INR is, the smaller $\alpha$ is, so the larger proportional coefficient of $\overline{\mathbf{R}}_{i+n}$ is needed. This fact motivates us to design $\beta$ using the interference power. As we know, the eigenvalue of the interference can be used to reflect the interference power, and thus the value $\lambda_{[1]}+\lambda_{[2]}+\cdots+\lambda_{[N-1]}$ can reflect the power

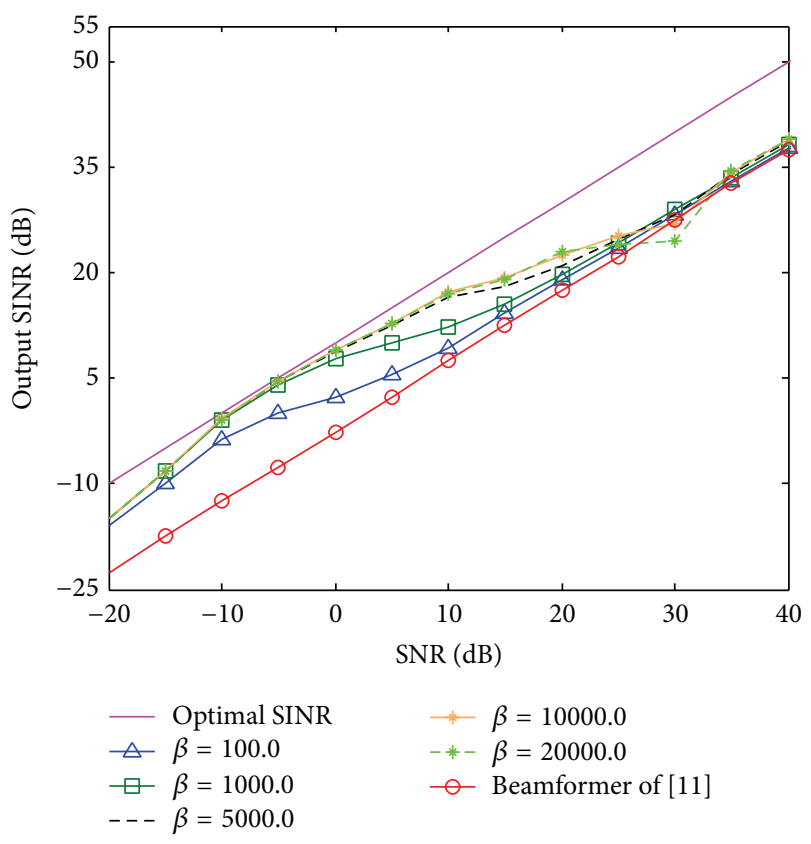

FIGURE 17: Output SINR versus the input SNR with different $\beta$ $(\mathrm{INR}=30 \mathrm{~dB})$.

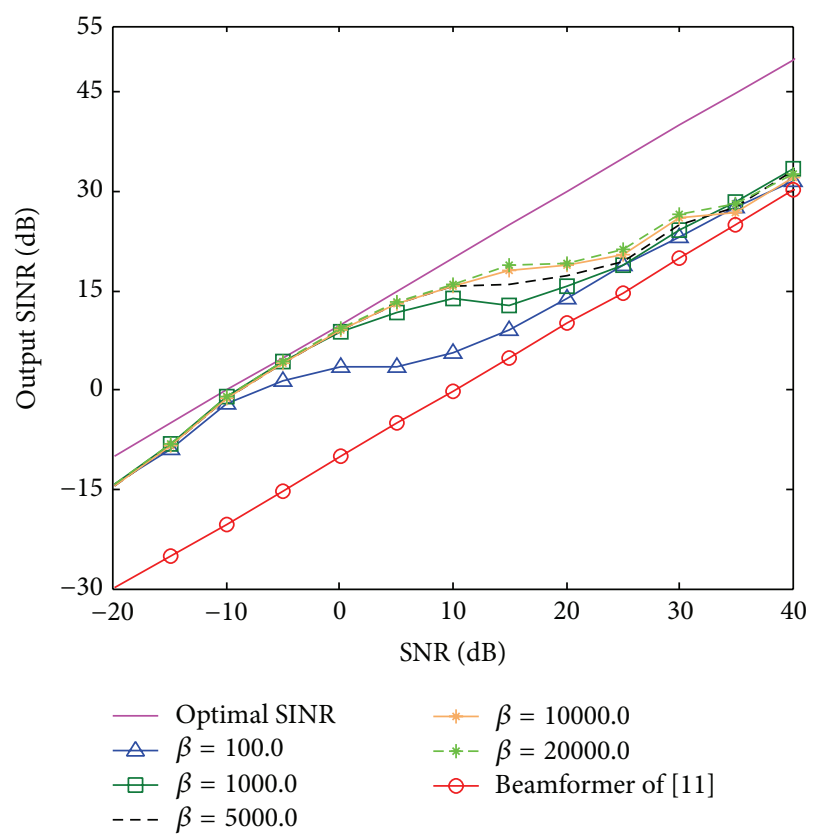

FIGURE 18: Output SINR versus the input SNR with different $\beta$ $(\mathrm{INR}=40 \mathrm{~dB})$.

of the interference (the power of the noise is small usually). We set the design parameter $\beta$ as

$$
\beta=\lambda_{[1]}+\lambda_{[2]}+\cdots+\lambda_{[N-1]} .
$$

It is worth noting that $\overline{\mathbf{R}}_{i+n}$ is the correlation matrix of the vectors which corresponds to the interference and noise. By taking the eigenvalues into consideration to design $\beta$ shown 


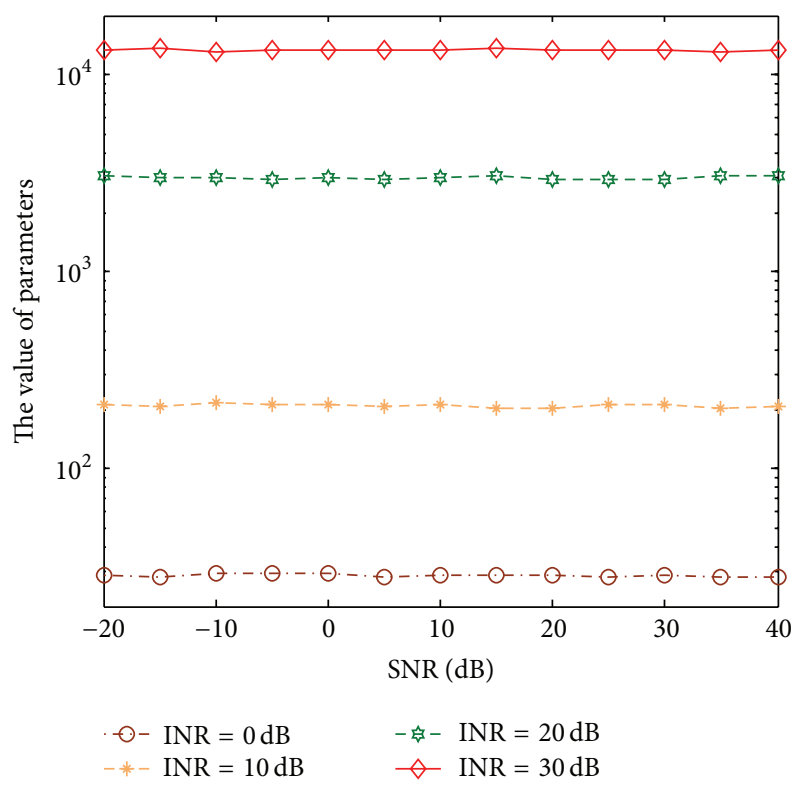

FIgURE 19: Values of $\beta$ versus the SNR.

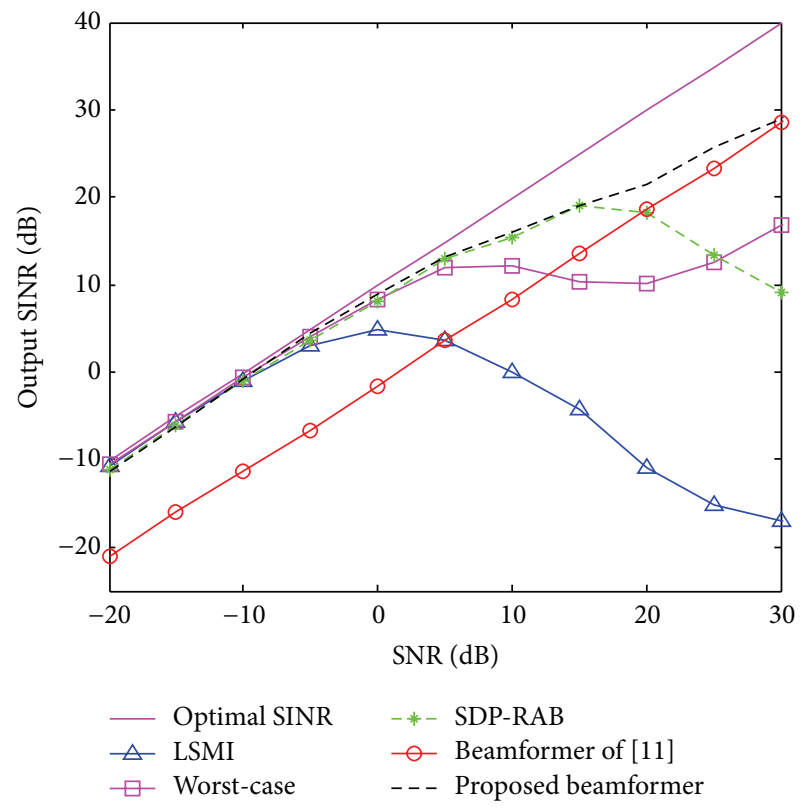

Figure 20: Output SINR versus the input SNR $(I N R=30 \mathrm{~dB})$.

in (23), the reconstructed IPN matrix will be more accurate. Figure 19 shows $\beta$ versus SNRs with various INRs.

We can observe from Figure 19 that the values of $\beta$ are almost the same in different SNRs with the fixed INR. The values of $\beta$ is positive to the INRs, which is consistent with our assumption. What is more, we can find that $\beta=3500$ when INR $=20 \mathrm{~dB}$ and $\beta=12000$ when INR $=30 \mathrm{~dB}$, which are consistent with the simulation results demonstrated in Figures 17 and 18.

Effectiveness of our designed method is verified on the basis of the same simulation experiments. Figure 20 shows the

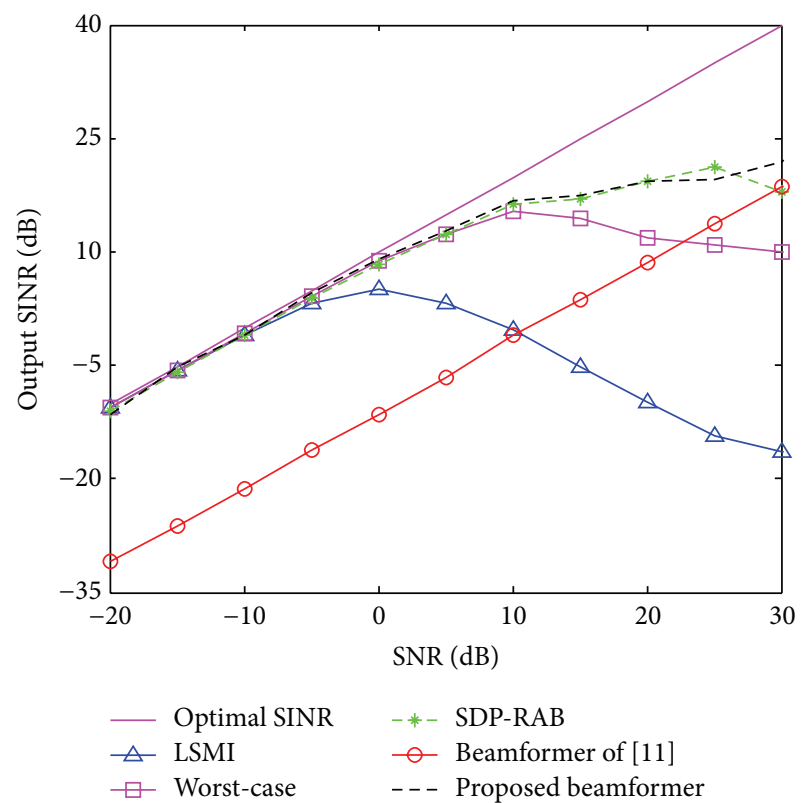

FIGURE 21: Output SINR versus the input SNR $(I N R=40 \mathrm{~dB})$.

output SINRs versus input SNR for INR $=30 \mathrm{~dB}$, and Figure 21 shows the output SINRs versus input SNRs for INR $=40 \mathrm{~dB}$.

We can see from Figures 20 and 21 that design parameters $\alpha$ and $\beta$ can obtain good performance in different situations. That means our method to design $\alpha$ and $\beta$ is correct and effective. In addition, to find a better approach to combine the two parts is also an interesting problem, which is an open topic for the people who are interested.

\section{Conclusion}

In order to further improve the robustness of adaptive beamformer, a high performance robust adaptive beamformer in the presence of various kinds of array imperfections was proposed and its performance was verified in detail. The proposed beamformer was realized via a modified method to reconstruct the IPN covariance matrix. The IPN covariance matrix comes from a weighted summation of two estimated covariance matrices and the proportion of the two estimated covariance matrices can be adjusted adaptively according to the input SNR and interference power. The simulation results demonstrate that the proposed beamformer can provide a superior performance against unknown arbitrary-type mismatches in a very large range of SNR.

\section{Competing Interests}

The authors declare that there are no competing interests regarding the publication of this paper.

\section{Acknowledgments}

This paper was supported by National Defense "973" Basic Research Development Program of China (no. 6131380101). 
This paper is also supported by Preresearch Fund of the 12th Five-Year Plan (no. 4010403020102 and no. 4010103020103) and the Fundamental Research Funds for the Central Universities (nos. HEUCFD1433 and HEUCF1508).

\section{References}

[1] H. L. Van Trees, Optimum Array Processing, Wiley, Hoboken, NJ, USA, 2002.

[2] J. Li and P. Stocia, Eds., Robust Adaptive Beamforming, John Wiley \& Sons, New York, NY, USA, 2005.

[3] W. Li, Y. Li, and W. Yu, "On adaptive beamforming for coherent interference suppression via virtual antenna array," Progress in Electromagnetics Research, vol. 125, pp. 165-184, 2012.

[4] X. Mao, W. Li, Y. Li, Y. Sun, and Z. Zhai, "Robust adaptive beamforming against signal steering vector mismatch and jammer motion," International Journal of Antennas and Propagation, vol. 2015, Article ID 780296, 12 pages, 2015.

[5] Z. L. Yu, M. H. Er, and W. Ser, "A novel adaptive beamformer based on semidefinite programming (SDP) with magnitude response constraints," IEEE Transactions on Antennas and Propagation, vol. 56, no. 5, pp. 1297-1307, 2008.

[6] K. Yang, Z. Zhao, and Q. H. Liu, "Robust adaptive beamforming against array calibration errors," Progress in Electromagnetics Research, vol. 140, pp. 341-351, 2013.

[7] L. Chang and C.-C. Yeh, "Performance of DMI and eigenspacebased beamformers," IEEE Transactions on Antennas and Propagation, vol. 40, no. 11, pp. 1336-1348, 1992.

[8] B. D. Carlson, "Covariance matrix estimation errors and diagonal loading in adaptive arrays," IEEE Transactions on Aerospace and Electronic Systems, vol. 24, no. 4, pp. 397-401, 1988.

[9] S. A. Vorobyov, A. B. Gershman, and Z.-Q. Luo, "Robust adaptive beamforming using worst-case performance optimization: a solution to the signal mismatch problem," IEEE Transactions on Signal Processing, vol. 51, no. 2, pp. 313-324, 2003.

[10] A. Khabbazibasmenj, S. A. Vorobyov, and A. Hassanien, "Robust adaptive beamforming based on steering vector estimation with as little as possible prior information," IEEE Transactions on Signal Processing, vol. 60, no. 6, pp. 2974-2987, 2012.

[11] Y. Gu and A. Leshem, "Robust adaptive beamforming based on interference covariance matrix reconstruction and steering vector estimation," IEEE Transactions on Signal Processing, vol. 60, no. 7, pp. 3881-3885, 2012.

[12] W. Jia, W. Jin, S. Zhou, and M. Yao, "Robust adaptive beamforming based on a new steering vector estimation algorithm," Signal Processing, vol. 93, no. 9, pp. 2539-2542, 2013.

[13] C. Gong, L. Huang, D. Xu, and Z. Ye, "Knowledge-aided robust adaptive beamforming with small snapshots," Electronics Letters, vol. 49, no. 20, pp. 1258-1259, 2013.

[14] L. Huang, J. Zhang, X. Xu, and Z. Ye, "Robust adaptive beamforming with a novel interference-plus-noise covariance matrix reconstruction method," IEEE Transactions on Signal Processing, vol. 63, no. 7, pp. 1643-1650, 2015.

[15] P. Stoica, J. Li, and X. Tan, "On spatial power spectrum and signal estimation using the Pisarenko framework," IEEE Transactions on Signal Processing, vol. 56, no. 10, part 2, pp. 5109-5119, 2008.

[16] M. Grant, S. Boyd, and Y. Y. Ye, "CVX: Matlab Software for Disciplined Convex Programming," 2014, http://cvxr.com/cvx/. 


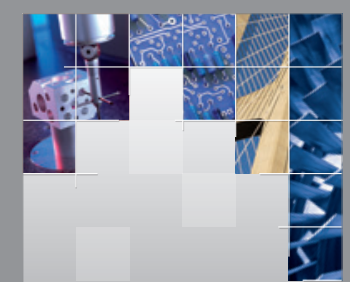

\section{Enfincering}
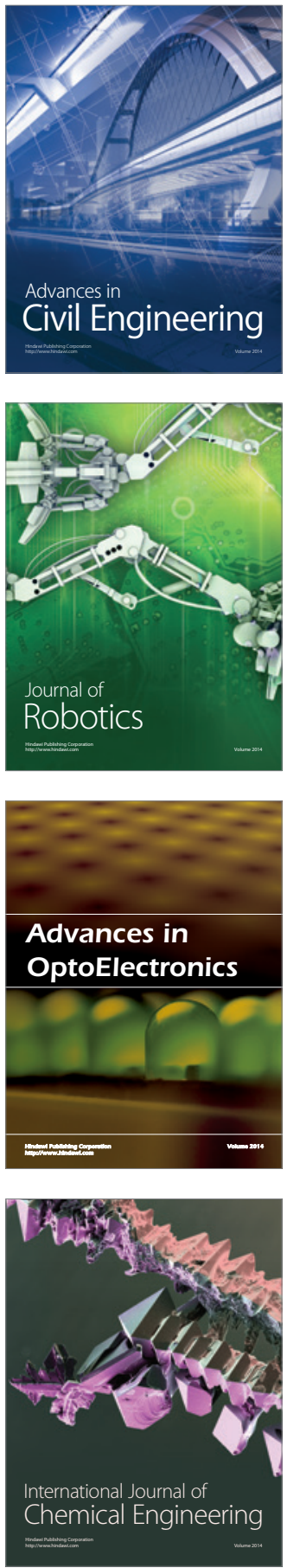

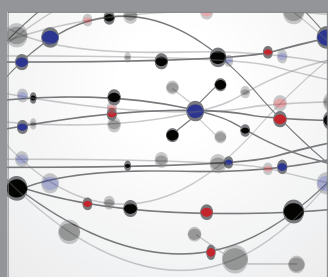

The Scientific World Journal

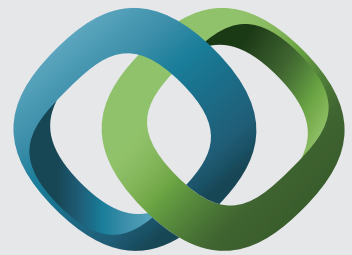

\section{Hindawi}

Submit your manuscripts at

http://www.hindawi.com
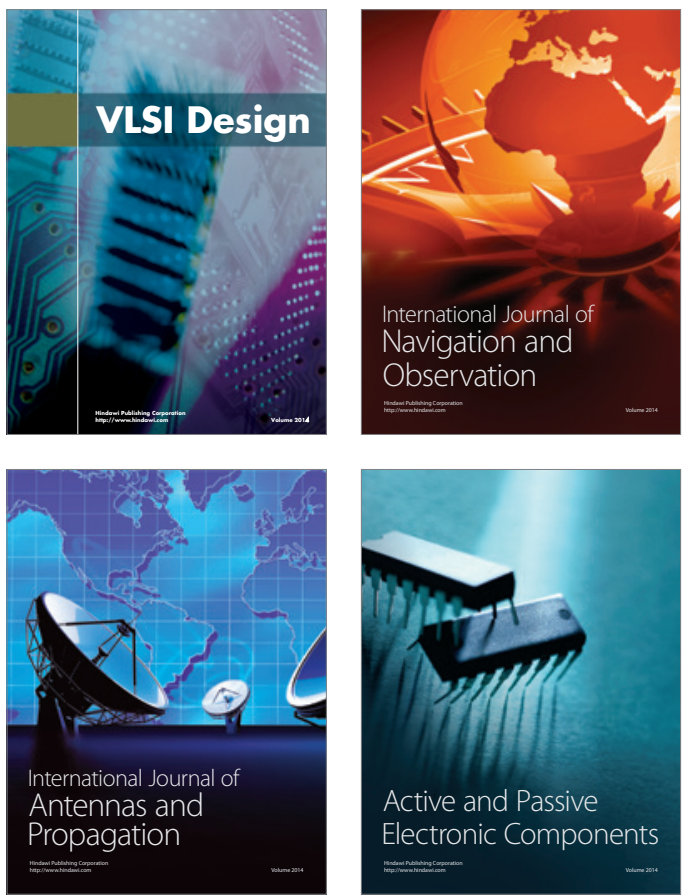
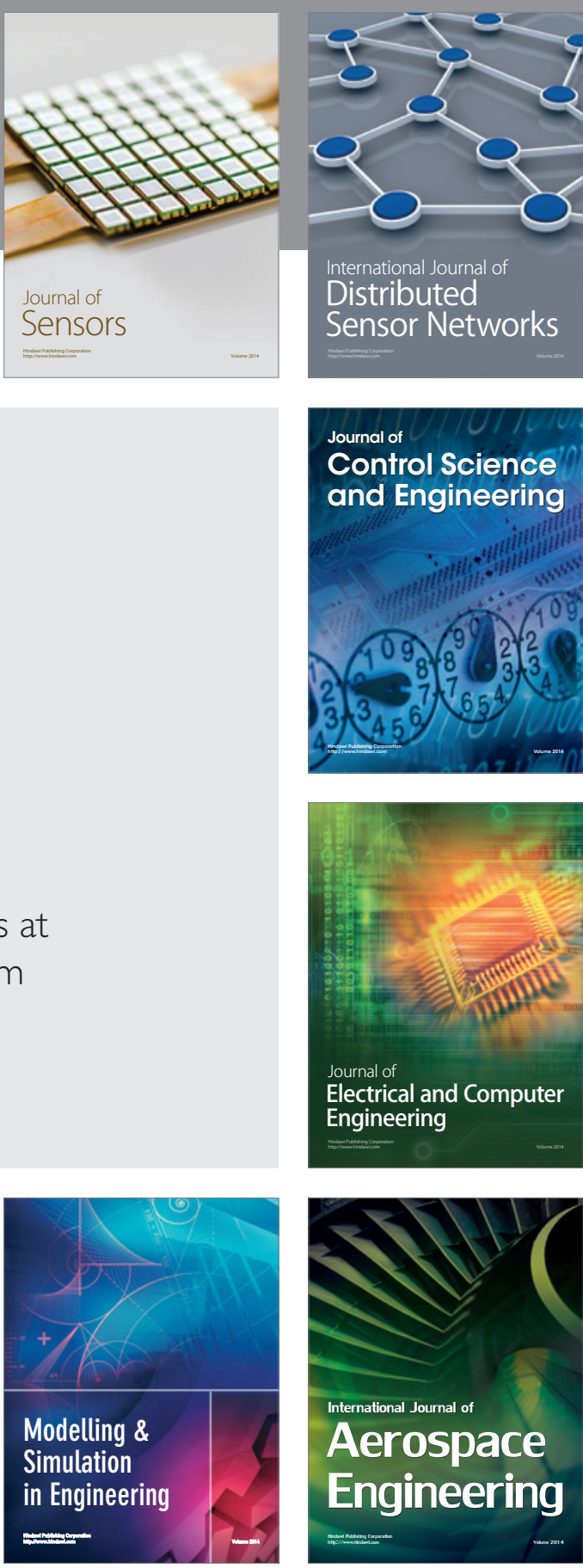

International Journal of

Distributed

Sensor Networks

Journal of

Control Science

and Engineering
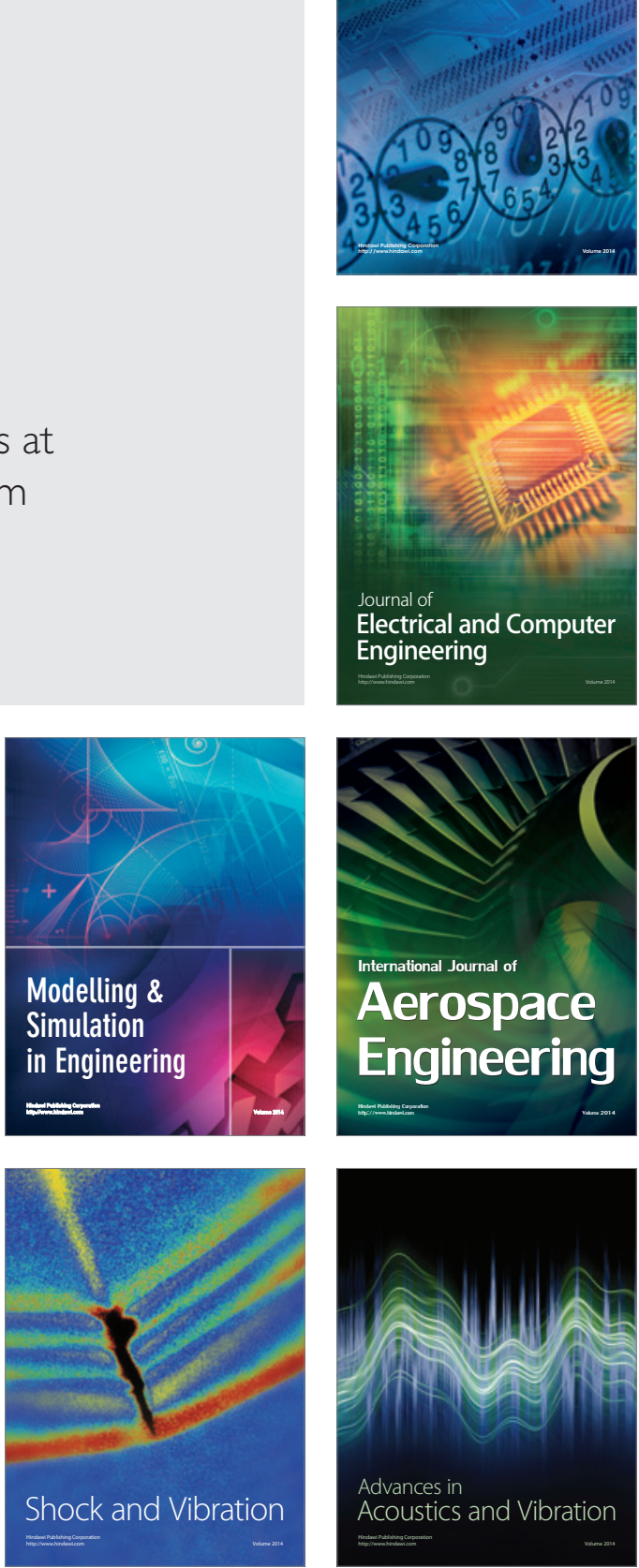